\title{
Mini-access branch-first total arch replacement and frozen elephant trunk procedure
}

\author{
Adam Chakos ${ }^{1,2}$, Tristan D. Yan ${ }^{1,2}$ \\ ${ }^{1}$ Department of Cardiothoracic Surgery, Royal Prince Alfred Hospital, The University of Sydney, Sydney, NSW, Australia; ${ }^{2}$ Department of Clinical \\ Medicine, Macquarie University, Sydney, NSW, Australia \\ Correspondence to: Tristan D. Yan. Professor, Head of Minimally Invasive \& Robotic Cardiothoracic Surgery Program, Department of Cardiothoracic \\ Surgery, Royal Prince Alfred Hospital, Sydney, NSW, Australia. Email: tristanyan@annalscts.com.
}

\begin{abstract}
Minimally-invasive surgery presents its own unique set of challenges, especially when applied to treatment of aortic arch disease. The key components of mini-access aortic arch surgery include safe circulatory control, adequate organ protection, and meticulous surgical technique. This article describes how to perform branchfirst total arch replacement with the frozen elephant trunk (FET) procedure for distal arch aneurysms. The strategy employed offers the advantages of mini-access surgery, as well as sound cerebral and systemic organ protection strategies.
\end{abstract}

Keywords: Frozen elephant trunk (FET); total arch replacement; minimally-invasive; mini-access; hemi-sternotomy

Submitted Apr 20, 2020. Accepted for publication May 21, 2020.

doi: 10.21037/acs-2020-fet-27

View this article at: http://dx.doi.org/10.21037/acs-2020-fet-27

\section{Introduction}

Total arch replacement with frozen elephant trunk (FET) is a technically demanding surgical procedure. Traditionally, total arch replacement was performed with interruption to the cerebral circulation under deep hypothermic circulatory arrest (1). This article details the operative steps for miniaccess branch-first total arch replacement with FET.

The branch-first technique uses a trifurcated graft to allow the epi aortic vessels to be debranched during systemic cooling, with no-or minimal interruption of cerebral perfusion $(2,3)$. After disconnecting the epi-aortic vessels, the entire aortic arch is fully exposed, enabling the distal aortic anastomosis to be performed comfortably. More importantly, the use of a zone 2 anastomosis avoids manipulation of the left recurrent laryngeal nerve and brings the distal arch anastomosis forward in the operative field. The combination of the branch-first technique and zone 2 arch anastomosis enables the procedure to be completed through a mini-access incision. Several key operative pitfalls and safeguards are highlighted in this article and particular attention has been paid to management of the left subclavian artery (LSCA).

\section{Patient selection}

All patients undergo transthoracic echocardiogram, coronary artery angiogram and computed tomography (CT) of the aorta to characterize the extent of the aortic aneurysm, atheromatous load, and degree of aortic calcification. It is important to assess the quality of the aorta at the bases of the epi-aortic vessels and the potential sites of aortic anastomoses. One key aspect of patient selection is whether absolute hemostatic anastomoses can be performed. Ideal candidates for mini-access arch replacement and FET are patients with distal arch aneurysms. In these cases, the epi-aortic vessels are usually anteriorized by the aneurysm and the quality of the zone 2 arch is usually satisfactory to ensure absolute hemostasis. Contraindications for the miniaccess approach include acute aortic syndrome, concomitant cardiac procedures (except aortic valve and root surgery), and redo surgery (Table 1).

\section{Preparation}

After intubation, radial and femoral arterial pressure monitoring lines, a central venous line, and pulmonary 
Table 1 Patient selection and pre-operative investigations for minimally-invasive FET and total arch replacement

\begin{tabular}{ll}
\hline Patient selection & Pre-operative investigations \\
\hline Indications & Transthoracic echocardiogram \pm transesophageal echocardiogram \\
Aortic arch aneurysm (preferably mid to distal arch) & Myocardial and valvular function \\
\hline Absolute contraindications & CT-aortogram \\
Dissection & Proximal and distal aortic tissue quality \\
Rupture & Atheroma load/calcification \\
Salvage surgery & Cannulation site, cross-clamp site \\
Relative contraindications & CT-coronary angiogram or coronary catheterisation \\
Poor LV function & Coronary disease assessment \\
Aberrant coronary anatomy & Coronary anomalies \\
Extremely large aneurysms & Carotid duplex ultrasound \\
Redo cardiac surgery & Carotid stenosis \\
Concomitant coronary artery bypass surgery & \\
\hline Concomitant non-aortic valvular surgery & \\
\hline
\end{tabular}

FET, frozen elephant trunk; CT, computed tomography; LV, left ventricular.

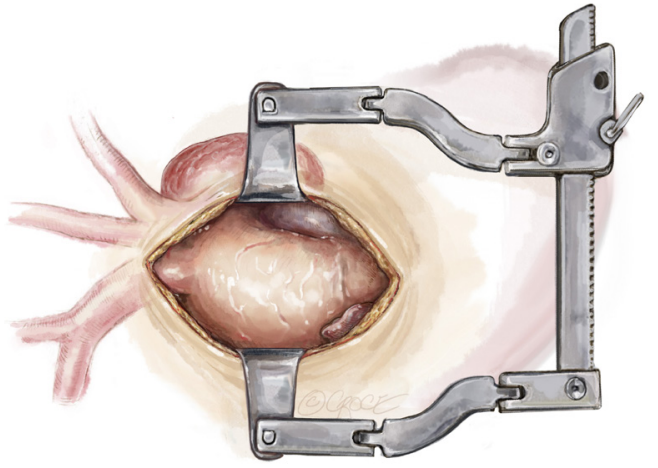

Figure 1 A mini-sternotomy is performed from the superior extent of the manubrium to the $3 \mathrm{rd}$ parasternal space. The thymic fat pad is mobilized and the innominate vein is slung with a vessel loop (not shown) to provide excellent visualization of the ascending aorta and arch vessels. Patients with distal arch aneurysms are best suited to this approach, as distal arch aneurysms tend to anteriorize the epi-aortic arch vessels.

arterial catheter are placed. The patient is positioned supine, with the neck slightly extended by placing a soft pad behind the shoulders. The suprasternal notch, sternomanubrial junction and intercostal spaces are identified with a marking pen. An alcohol-based iodine solution is painted on the skin and evaporated to dryness before the patient is draped widely to allow access to the groins and the right and left subclavian arteries. Prior to incision, appropriate prophylactic antibiotics are administered.

\section{Mini-access incision}

A midline skin incision is performed from the sternal notch to the level of the third intercostal space. The incision is usually $7 \mathrm{~cm}$ in length depending on the body size of the patient. It is developed through the subcutaneous fat onto the body of the sternum using diathermy. A limited subcutaneous flap is developed with a diathermy handpiece. This flap is extended laterally to the limits of the sternum on the left side. The supra-sternal bridging vein is clipped and cut. A 14 Fr Jackson Pratt drain (Cardinal Health, McGaw Park, IL, USA) is inserted inferior to the incision in the pre-sternal plane, coming out through the skin at the tip of the xiphoid. This is used for carbon dioxide inflow during the case to prevent air embolism and as a subcutaneous drain at the completion of the operation.

A mini-sternotomy is performed using a hand-held electrical saw from the superior extent of the manubrium. The division is terminated at the left third para-sternal space (Figure 1). Hemostasis is achieved by applying a small 


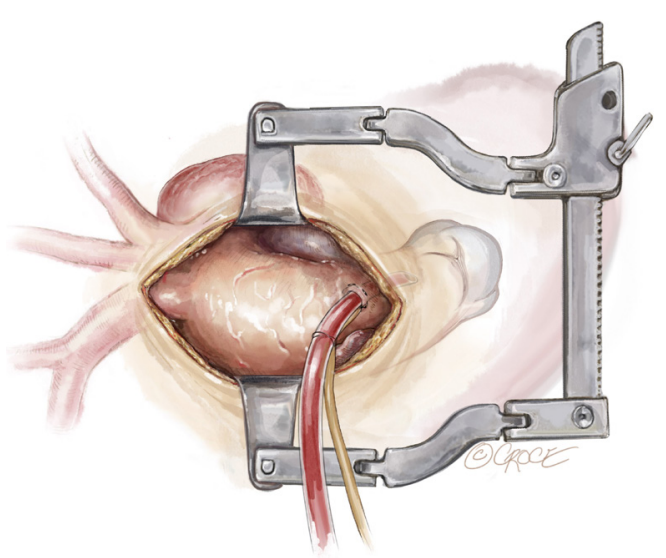

Figure 2 As distal arch aneurysms usually contain mobile atheroma, the aortic cannula should be directed toward the aortic root to avoid "sandblasting" atheroma and causing distal embolization.

amount of wax to the bone marrow. A mini-access sternal retractor is inserted and opened gradually to expose the anterior mediastinum.

\section{Extra-pericardial dissection}

The thymic fat pad is completely mobilized to the innominate vein superiorly and excised to provide visualization of the great vessels. Extra-pericardial dissection of brown fat around the innominate vein and epi-aortic vessels is completed using a $3 \mathrm{~mm}$ ball-tipped diathermy to delineate each arch vessel. The innominate artery, left common carotid artery (LCCA) and LSCA are then each slung with vessel loops for safety and control.

\section{Intrapericardial dissection}

With the epi-aortic arch vessels delineated and controlled, the pericardium is opened by making an inverse " $\mathrm{T}$ " incision from the distal ascending aorta to the level of right atrial appendage. The aortic root is anteriorized to provide optimal access by placing three traction sutures in each side of the pericardium and hitching them to the skin. To avoid hypotension as a result of vagal stimulation and impedance of preload, these traction sutures should be hitched up gradually and the anesthetist should be informed before doing so. The mini-access sternal retractor is then re-inserted and opened gradually. With the operative field prepared, heparin is administered to an activated clotting time of greater than 450 seconds in preparation for cannulation and initialization of cardiopulmonary bypass (CPB).

\section{Cardiopulmonary bypass}

A vacuum-assist device on the CPB circuit is used to maximize venous drainage. Peripheral venous cannulation is established first by using the Seldinger technique. After puncture of a femoral vein, a guide wire is passed up to the superior vena cava. The position of the wire is confirmed with transesophageal echocardiography (TEE) using a bicaval view. The femoral vein puncture site is then progressively dilated and a 25 Fr multi-stage venous cannula (Maquet Getinge Group, Rastatt, Germany) is introduced. The tapered tip insert is not advanced further once it enters the right atrium. Only the venous cannula itself is now advanced forward over the insert, strictly under TEE guidance. It is essential that the cannula tip is placed in the superior vena cava to ensure satisfactory bicaval venous return. The venous cannula is subsequently connected to the $\mathrm{CPB}$ circuit.

Before placing the aortic cannula, an intraoperative epi-aortic ultrasound should be performed. It is crucial that the arterial cannula is directed away from the distal arch aneurysm, which may be typically lined with mobile atheroma. The cannula tip should be directed towards the aortic root to avoid a "sandblast" effect from the arterial cannula, which could dislodge atheroma and cause distal embolization. With the above assessment made, a purse string is placed in the proximal ascending aorta and a 22 French arterial cannula inserted towards the aortic valve and snared down (Figure 2). TEE should be used to ensure that the patient does not have significant aortic regurgitation and the arterial cannula tip is not crossing the aortic valve.

\section{Innominate artery debranching}

As the patient is cooling on CPB towards 20 degrees, debranching of the epi-aortic vessels is performed, starting with clamping the innominate artery and oversewing the remnant stump with two layers of 4-0 Prolene sutures (Figure 3). The central limb of a trifurcated graft is then anastomosed in an end-to-end to fashion to the innominate artery using a 5-0 running Prolene suture (Figure 4). During this period, the brain is perfused via the LCCA and the LSCA.

After completion of the innominate artery debranching, a separate perfusion cannula from the $\mathrm{CPB}$ circuit is secured on the perfusion limb of the trifurcated graft, the graft is de-aired, and a clamp is placed proximal to the perfusion 


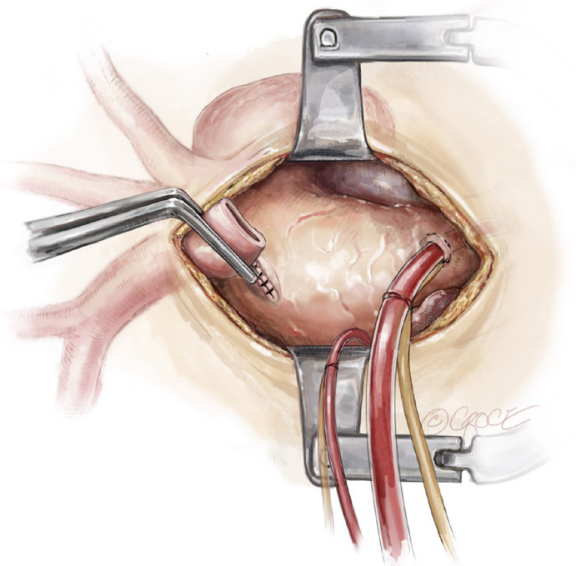

Figure 3 During cooling to deep hypothermic circulatory arrest, the epi-aortic arch vessels are detached sequentially. The innominate artery is clamped first, divided, and the remnant stump is oversewn with 4-0 Prolene sutures. At this point, the brain is perfused by the LCCA and LSCA. LCCA, left common carotid artery; LSCA, left subclavian artery.

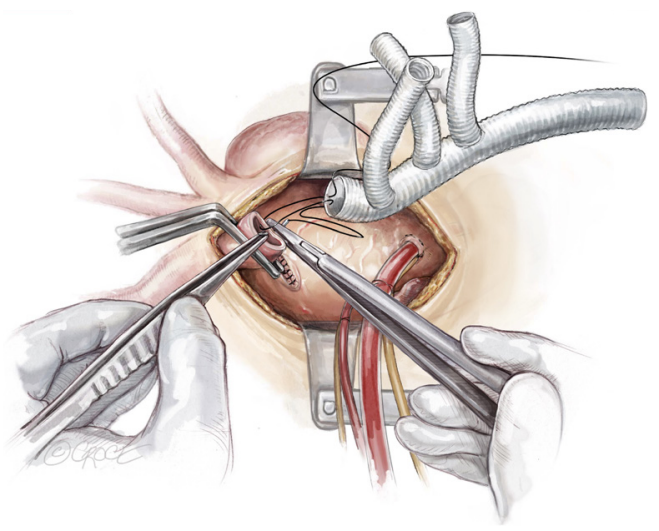

Figure 4 After division of the innominate artery, end-to-end anastomosis to the main limb of the trifurcated graft is completed using a 5-0 running Prolene suture.

limb. Perfusion of the right-sided cerebral circulation is then commenced via the innominate artery.

\section{Left common carotid artery debranching}

After debranching the innominate artery, attention is turned to the LCCA, which is clamped at the base and the remnant stump oversewn with two layers of 4-0 Prolene sutures. The second limb of the trifurcated graft is then anastomosed end-to-end to the LCCA with a 5-0 running Prolene suture

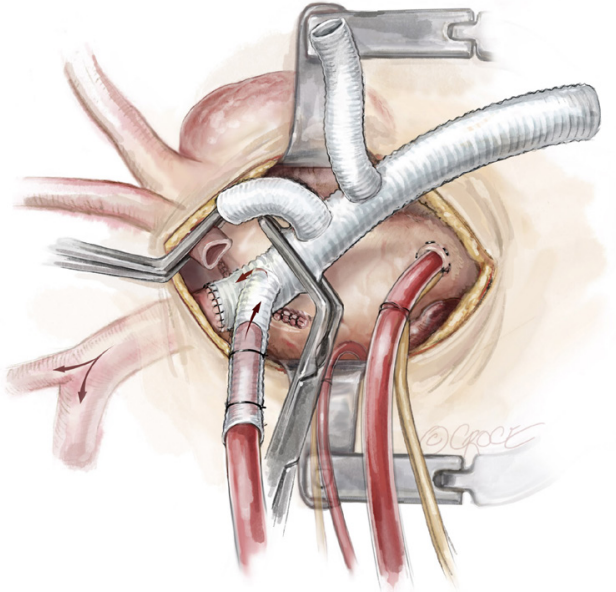

Figure 5 With the innominate artery perfused via the perfusion limb of the trifurcated graft, the LCCA is clamped and divided. The remnant stump is oversewn with two layers of 4-0 Prolene suture. The LCCA is anastomosed end-to-end with the trifurcated graft using 5-0 Prolene sutures. The arrow indicates direction of blood inflow from cardiopulmonary bypass circuit. LCCA, left common carotid artery.

(Figure 5). The cerebral circulation is maintained via the innominate artery and the LSCA during this period. After completion of the LCCA debranching, the clamp at the base of the LCCA is released to de-air the graft, and the graft clamp is moved to between the LCCA and LSCA limbs of the trifurcated graft. Bilateral cerebral perfusion is then re-established.

\section{Left subclavian artery management strategies}

With the brain perfused bilaterally via the trifurcated graft, the LSCA is then managed according to aortic pathology, surgical access, and surgeon preference. The key is to ensure absolute hemostasis, as this anastomosis is usually deep in the chest and more difficult to control. The advantage of having debranched the innominate artery and the LCCA by this time is that it readily exposes the LSCA. In addition, due to progressive systemic cooling approaching deep hypothermia, the patient is able to tolerate a period of low systemic blood flow which allows the aortic arch to be retracted downwards slightly to increase the accessibility of the LSCA.

\section{Left subclavian artery debranching}

The first operative strategy to manage the LSCA is routine 


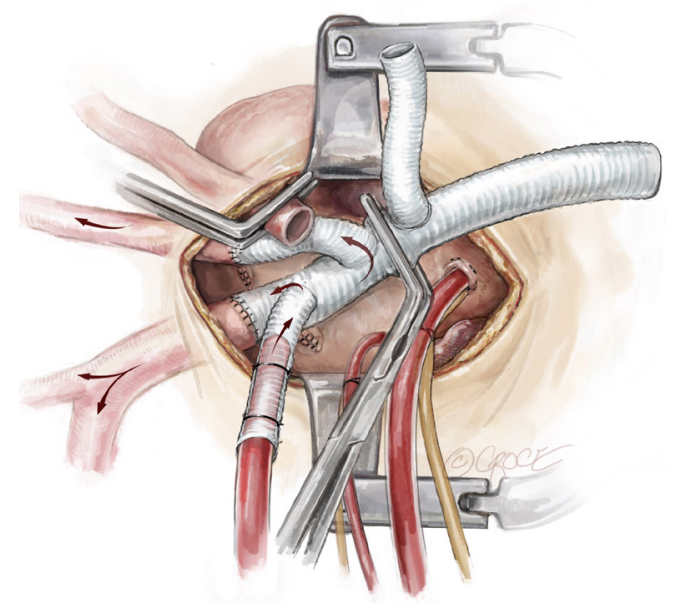

Figure 6 The LSCA is clamped at the base and the remnant stump is oversewn with two layers of 4-0 running Prolene sutures. Perfusion of the brain is via the perfusion limb of the trifurcated graft through the innominate and LCCA. The arrow indicates direction of blood inflow from cardiopulmonary bypass circuit. LCCA, left common carotid artery; LSCA, left subclavian artery.

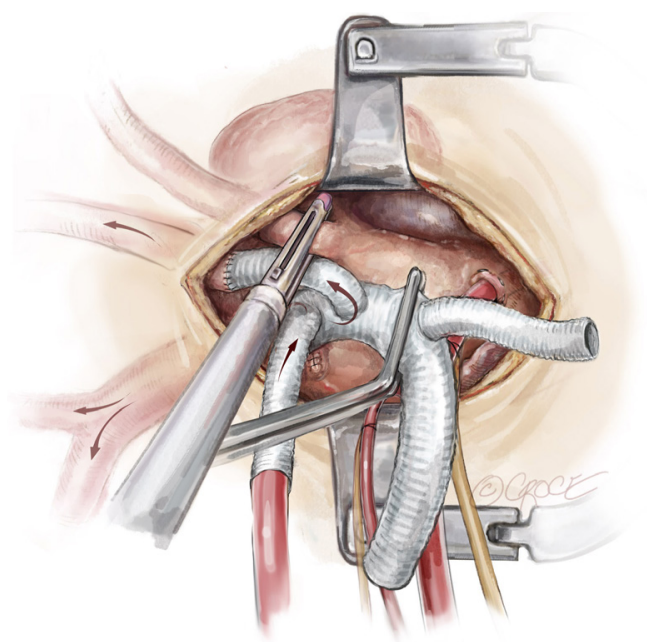

Figure 7 For difficult-to-access left subclavian arteries, a vascular stapler can be used to ligate the LSCA, however, care must be taken not to injure the left recurrent laryngeal nerve. The arrow indicates direction of blood inflow from cardiopulmonary bypass circuit. LSCA, left subclavian artery.

debranching, when access to the LSCA is adequate. This is the case especially when the aneurysm is in the distal arch, pushing the LSCA forward. The LSCA is clamped at the base and the remnant stump is oversewn with two layers of 4-0 Prolene sutures (Figure 6). The third limb of a trifurcated graft is then anastomosed in an end-to-end fashion to the LSCA with a 5-0 running Prolene suture. The cerebral circulation is maintained via the perfusion limb to the innominate artery and the LCCA during this period. After completion of the LSCA debranching, the clamp at the base of the LSCA is released to de-air the graft, the graft clamp is moved proximal to the LSCA limb of the trifurcated graft, and full cerebral perfusion is re-established via the trifurcated graft.

\section{Left subclavian artery extra-anatomic bypass}

Where the LSCA is too deep in the chest or the base of the LSCA is severely calcified, direct end-to-end anastomosis would be deemed difficult or unsafe. Under these circumstances, extra-anatomic bypass strategies should be considered. These can be performed via a supraclavicular incision, allowing placement of an interposition graft between the LSCA and LCCA.

Another surgical approach is to make a $2 \mathrm{~cm}$ infraclavicular incision to expose a short segment of the left axillary artery under the pectoralis minor muscle. An $8 \mathrm{~mm}$ graft is anastomosed to the left axillary artery in an endto-side fashion, using a 5-0 Prolene suture. This graft is then tunneled inferiorly between the pectoralis major and minor muscles and dropped into the left thoracic cavity via the second intercostal space. It is then brought through to the mediastinum via a small incision in the mediastinal pleura, in readiness for re-implantation to the last limb of the trifurcated graft during the later rewarming phase of the operation. The base of the subclavian artery can be ligated with two heavy silk ties or stapled off with a vascular stapler at its base. Care must be taken not to ligate or staple calcified LSCA and the adjacent left recurrent laryngeal nerve (Figure 7).

\section{Frozen elephant trunk stent graft deployment}

After having debranched all three epi-aortic vessels, the patient's systemic temperature has typically reached the 20 degrees Celsius target. An aortic cross clamp is applied proximal to the aortic cannula and diastolic cardiac arrest is achieved with antegrade cardioplegia delivery. The patient is positioned head down, the blood emptied into the CPB reservoir, and the aorta distal to the cross clamp is opened longitudinally to zone 2 of the aortic arch. A drop sucker is placed into the mid-distal arch to evacuate blood and 


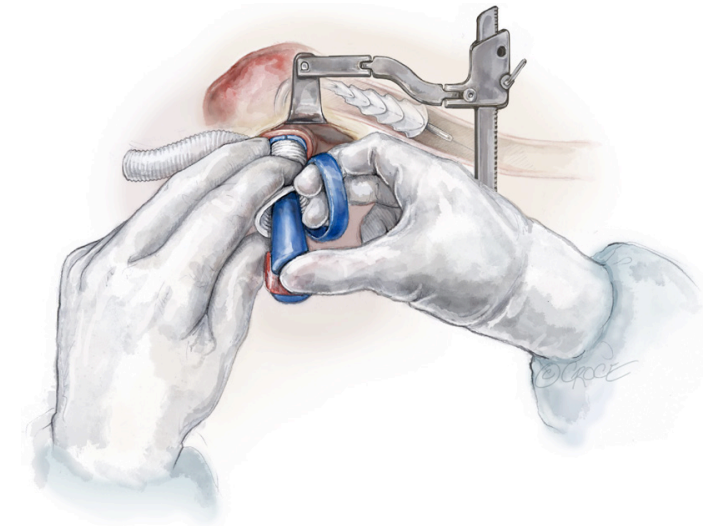

Figure 8 The Thoraflex Hybrid graft (Terumo Aortic, Inchinnan, UK) is bent to conform to the curvature of the descending aorta before being inserted into the distal arch. In aortic dissection cases, it is inserted over a Linderquist wire placed in the true lumen. With the handle stabilising the graft, the sheath is then retracted back through the splitter to deploy the self-expanding stent.

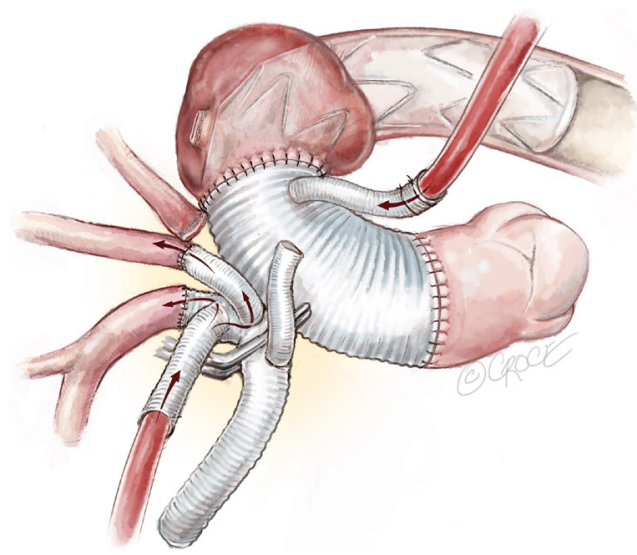

Figure 9 Completed zone 2 and proximal aortic anastomoses. Systemic perfusion is via the FET side arm and cerebral perfusion via the trifurcated graft perfusion limb. The arrow indicates direction of blood inflow from cardiopulmonary bypass circuit. FET, frozen elephant trunk.

facilitate inspection of the aortic aneurysm and its thrombus load. In an ideal situation, the atheroma should not be touched (the "no touch" technique), rather, they should be excluded from the systemic circulation by coverage with the FET. However, extremely loose atheroma may be removed with a metal sucker at this stage to avoid embolization with the introduction of the FET.

A Thoraflex hybrid ${ }^{\mathrm{TM}}$ graft (Terumo Aortic, Inchinnan,
UK) is selected for antegrade deployment into the proximal descending thoracic aorta. The stented portion of the Thoraflex hybrid graft is bent slightly to conform to the curvature of the descending thoracic aorta and is guided down over a stiff Linderquist wire in the true lumen for aortic dissection cases. The handle is used to stabilize the graft in position. The sheath is retracted back through the splitter to deploy the self-expandable stent (Figure 8 ). The splitter is then removed and the sewing collar is freed. Finally, the stent tip is freed by removing the central locking pin through the device handle and the delivery system is released from the hybrid graft.

The distal aortic reconstruction is performed with the sewing collar of the Thoraflex graft to the zone 2 arch, using a 3-0 running Prolene suture. This anastomosis is then reinforced with interrupted pledgeted 3-0 Prolene sutures or a second layer of 3-0 running Prolene suture with a circumferential Teflon felt strip.

After completion of the zone 2 anastomosis, a perfusion cannula is inserted to the side-branch of the Thoraflex graft for antegrade distal organ perfusion. The graft is deaired and the distal aortic reconstruction is inspected for hemostasis. Systemic rewarming is commenced. FET stent expansion and isolation of the diseased aorta should be confirmed by TEE at this stage.

Proximal aortic reconstruction is achieved by anastomosing the proximal end of the Thoraflex graft to the sinotubular junction using a 3-0 running Prolene suture. After deairing, the cross clamp is released and the heart is re-perfused via the perfusion limb of the Thoraflex graft (Figure 9).

\section{Reimplantation of trifurcated graft}

With systemic perfusion via the Thoraflex graft sidearm and cerebral perfusion via the trifurcated graft, an appropriate location for trifurcated graft reimplantation is selected on the ascending aortic graft to prevent kinking or twisting of the conduit. A side-biting clamp is then applied to the ascending aortic graft and the proximal end of the trifurcated graft reimplanted in an end-to-side fashion with 4-0 running Prolene sutures (Figure 10).

If an extra-anatomical bypass of the LSCA has been planned, the debranched left axillary artery graft is tunneled between the pectoralis major and minor muscles and brought through the left thoracic cavity via left second intercostal space. It is then anastomosed with the third 


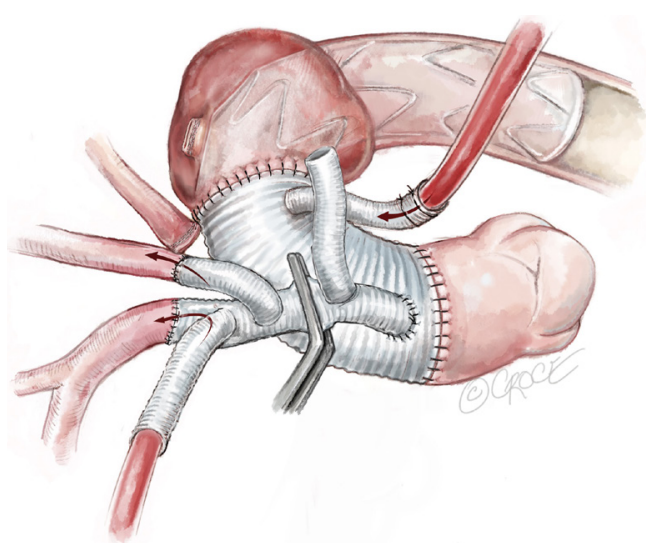

Figure 10 Attachment of the trifurcated graft to the proximal aortic graft is performed using continuous 4-0 Prolene sutures with the aid of a side-biting clamp (not shown). The final limb of the trifurcated graft is then free for attachment to the LSCA in extraanatomic bypass cases. The arrow indicates direction of blood inflow from cardiopulmonary bypass circuit. LSCA, left subclavian artery.

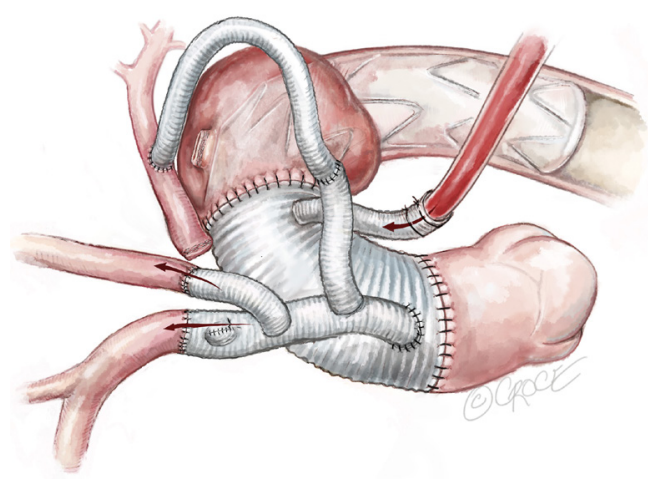

Figure 11 Completion of innominate artery and LCCA debranching and extra-anatomic bypass of the LSCA. Whole body perfusion is provided by the Thoraflex graft perfusion limb while the patient is re-warmed to 37 degrees Celsius. The arrow indicates direction of blood inflow from cardiopulmonary bypass circuit. LCCA, left common carotid artery; LSCA, left subclavian artery.

limb of the trifurcated graft during systemic rewarming (Figure 11). The epi-aortic vessels are now perfused completely via the Thorflex graft. The perfusion limb of the trifurcated graft is ligated with heavy silk ties. Re-warming of the patient towards 37 degrees Celsius continues and a thorough hemostatic checked is performed.

\section{Completion}

A bi-polar temporary pacing wire is inserted in the epicardium over the right ventricular outflow tract, which is brought out to the skin over the supra-sternal notch. Two $28 \mathrm{Fr}$ soft drains are inserted and brought out below the xiphoid cartilage in the pre-pericardial space. Hemostasis is carefully checked and the patient is weaned from CPB. Protamine is given to reverse the Heparin effect. Topical Floseal Hemostatatic Matrix (Baxter Healthcare, Zurich, Switzerland) is applied around the anastomotic sites. The surgical site is packed with small gauze sponges for a 10-minute hemostatic pause. Once hemostasis is deemed satisfactory, three stainless steel wires are used to approximate the sternum. No. 1 Vicryl suture is used to close the fascia and the subcutaneous fat. The skin is closed with a 5-0 Monocryl subcuticular suture. This completes the mini-access branch-first total arch replacement and FET procedure.

\section{Comment}

For selected patients with mid- and distal aortic arch aneurysms, the mini-access branch-first total replacement and FET with zone 2 anastomosis is a valuable addition to the aortic surgeon's armamentarium. An important aspect of the minimal access set up is that the division of the upper part of the sternum is terminated to the left para-sternal space, not to the right side. The main advantage of doing a mini-sternotomy to the left is increasing exposure of the aortic arch and access to the left subclavian artery, which is crucial in determining the success of this operation. In order to provide adequate surgical accessibility, it is important to pull firmly on the pericardial traction sutures on the right side, which anteriorizes the ascending aorta and rotates the axis of the aortic arch towards the operator. All thymic fat pad and epi-aortic fat pads must be completely removed. These simple steps provide excellent exposure of the epiaortic vessels and the aortic arch through a mini-access incision.

As a general rule when dealing with distal arch or proximal descending aortic aneurysms, retrograde perfusion via the femoral artery is not advised. It is preferable to have central arterial cannulation whenever possible to provide adequate antegrade systemic perfusion and avoid potential retrograde embolization and vascular complications that may be associated with peripheral cannulation. In this cohort of patients where atheromatous disease burden is 
significant in the distal arch, the arterial cannula tip should be pointed towards the aortic valve to avoid a "sandblast" effect from the arterial inflow cannula. Epi-aortic ultrasound and intraoperative TEE are important imaging modalities to confirm the presence of the atheroma and the ideal position of the cannula tip.

The advantages of the branch-first approach include continuous cerebral perfusion throughout the case, efficiently utilizing operative time during systemic cooling, and significantly reducing the risk of cerebral air embolism, as the cerebral circulation is in a "closed" system during debranching. More importantly in mini-access arch surgery, this approach improves exposure to the next epi-aortic vessel after previous one is debranched.

By completing the aortic anastomosis at zone 2, potential injury to the left recurrent laryngeal nerve and manipulation of the distal arch is minimized. The distal arch is commonly associated with large atheroma burden and poor tissue quality (3). This also allows the distal arch anastomosis to be technically feasible through a mini access approach.

Another challenging aspect of aortic arch surgery is the management of the LSCA. In cases where the LSCA origin is deep in the chest or severely calcified, the use of an extra-anatomical bypass should be considered as a valid approach. This may take the form of a left common carotidleft subclavian bypass, or an extra-anatomic bypass graft from the left axillary artery to the trifurcated graft via the left chest. The less common but promising option of hybrid endovascular debranching devices offer another alternative option for the management of the LSCA (or even all three arch branches) (4). VORTEC (Viabahn Open Rebranching Technique) uses Viabahn or Hemobahn stent-grafts (W. L. Gore and Associates, Flagstaff, Ariz) and can potentially simplify the distal LSCA anastomosis deep in the chest, since debranching of the vessel is achieved entirely with an endovascular stent distally_-without the need to suture branches (5).

In the strategy presented, the fundamental principles of a traditional aortic root replacement are respected and it must be emphasized that a meticulous surgical technique to ensure absolute hemostasis is of utmost importance in minimally invasive surgery. A complete aortic repair via a minimal-access incision and successful treatment in selected patients with aortic arch aneurysm is feasible.

\section{Acknowledgments}

None.

\section{Footnote}

Conflicts of Interest: The authors have no conflicts of interest to declare.

Open Access Statement: This is an Open Access article distributed in accordance with the Creative Commons Attribution-NonCommercial-NoDerivs 4.0 International License (CC BY-NC-ND 4.0), which permits the noncommercial replication and distribution of the article with the strict proviso that no changes or edits are made and the original work is properly cited (including links to both the formal publication through the relevant DOI and the license). See: https://creativecommons.org/licenses/by-nc-nd/4.0/.

\section{References}

1. Coselli JS, Green SY. Evolution of aortic arch repair. Tex Heart Inst J 2009;36:435-7.

2. Matalanis G, Galvin SD. "Branch-first" continuous perfusion aortic arch replacement and its role in intra-operative cerebral protection. Ann Cardiothorac Surg 2013;2:194-201.

3. Galvin SD, Perera NK, Matalanis G. Surgical management of acute type A aortic dissection: branch-first arch replacement with total aortic repair. Ann Cardiothorac Surg 2016;5:236-44.

4. Desai ND, Hoedt A, Wang G, et al. Simplifying aortic arch surgery: open zone 2 arch with single branched thoracic endovascular aortic repair completion. Ann Cardiothorac Surg 2018;7:351-6.

5. Donas KP, Rancic Z, Lachat M, et al. Novel sutureless telescoping anastomosis revascularization technique of supra-aortic vessels to simplify combined open endovascular procedures in the treatment of aortic arch pathologies. J Vasc Surg 2010;51:836-41.

Cite this article as: Chakos A, Yan TD. Mini-access branchfirst total arch replacement and frozen elephant trunk procedure. Ann Cardiothorac Surg 2020;9(3):236-243. doi: 10.21037/acs-2020-fet-27 